\title{
An Fuzzy Trust Based Energy Aware Multipath Secure Data Collection in WSN
}

\author{
Y. Chitti Babu ${ }^{1 *}$, Ch. Anuradha ${ }^{2}$, P. Sri Rama Chandra Murty ${ }^{3}$

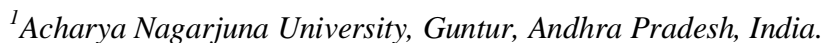 \\ ${ }^{2}$ V.R. Siddhartha Engineering College, Vijayawada, Andhra Pradesh, India. \\ ${ }^{3}$ Acharya Nagarjuna University, Guntur, Andhra Pradesh, India \\ *Corresponding author E-mail:chitti510@gmail.com
}

\begin{abstract}
A Wireless Sensor Network (WSN) is an array of radar nodules that energetically organize itself into a cable-less grid without utilizing any prevailing arrangement. One of the significant issues in WSNs is the drive's intake, whereby the grid's lifespan is reliant on this aspect. Security is another significant issues in cable-less radar grids, because of absence of wire/cable besides resource constraint's nature. Reliance model shows a key role in fortifying the radar grids by recognizing the egotistical, malevolent and give-in nodules and separating them from message grid. Directed Random Propagation (DRP) operates two-hop vicinity data to enhance the circulation competence, leading to a reduced pack capture possibility. In this study, Shuffled Frog Leaping Algorithm (SFLA) is applied to augment ill-defined faith centered drive conscious multiple route locked data group in WSN.
\end{abstract}

Keywords: Wireless Sensor Network (WSN), energy consumption, Directed Random Propagation (DRP) and Shuffled Frog Leaping Algorithm (SFLA).

\section{Introduction}

Wireless Sensor Networks is mainly exploited for data gathering besides combination. Data gathering is described as the planned assimilation of known data from manifold radars eventually communicated to the base station for dispensation. However, the data produced from nearby radar nodules is obstinately irrelevant and greatly consistent. In such scenarios, the radar nodules can convey data to a native antenna or allotted head which merges the data from all the radar nodules residing in its associativity and conveys the brief pack to the base station inevitably minimizing the total pack communication thus, saving frequency range and drive. This can be achieved by data combination [1] which can be described as the supervisory attitude to merge the detected data from many radar nodules with removal of irrelevance and deliver shared information to the base station.

Directing is one of the significant hitches in the WSN and is one of the most stimulating study areas in the communiqué grid. Directing the information is thus executed natively and step-bystep. Each nodule which is self-governing in conclusions in other nodules and its past decision makes a new directing decision. Directing usually direction centered on the directing records existing in each nodule's Routing Table (RT) to several ends in the grid. The RT is a table that is stored in nodule memory lists, directs to special nodules. Of late, processes act contrarily to locate the direction between the sender and receiver; more processes only find a direction and some directing processes find a manifold path. The multi-path direction processes waste additional grid assets due to more communication broadcast as contrasting to the single pathway processes $[2,3]$.

The important purpose of the faith prototypical [4] is to enhance the complete grid's functioning by overseeing the nodule's manners, gathering and computing the faith's worth of the nodules, minimizing the power of outbreaks and guaranteeing the grid's consistency on data collection and dispensation. Reliance is expressed as the two-fold connection happening in question and purpose. The reliance managing method relies on the background testimony of entity's manners or the interface conduct. The testimony is used to compute a trust's worth. The trust's worth enables a forecast of upcoming manners and governs the entity's subsequent action. The assessment of the trust's worth comprises nodule faith, connection's faith and facility's faith. This approach enables the faith's arrangement mechanism to be real in enhancing the safety of the grid in an open milieu.

Ambiguous Faith Forecasting Directing was planned in [5] with the under mentioned goals:

To reduce the drive's intake by evading unrestrained style of working for fellow citizen by overseeing and minimizing the quantum of references gained from fellow citizen to calculate unintended faith.

To minimize pack's cost by classifying and removing malevolent nodules by forecasting faith. The faith of an adjacent nodule is forecasted depending on straight faith, quantum of faith's variations besides inconsistency of recommendation.

Augmentation of cable-less radar grids comprise countless compromises, for example, lesser communication drive vs. lengthier communication period, ad hoc vs. straight communiqué, calculation vs. communique etc. Uncertain judgement is thriving right for implementations having incompatible necessities. Besides, in WSN, the drive's system varies greatly with the kind of radar nodule application program, using uncertain judgement has the benefit of being humbly adjustable to such alterations.

Augmentation methods wishes to locate the finest feasible answer within the restrictions described for an assumed issue. The augmentation processes can either be free-will or randomly determined in nature. The SFLA is a group intellect empirical which pools the benefits of element group and imitated culture procedures. It is an inhabitants-centered supportive higher-level 
procedure designed to find, generate or select with robust calculation function and decent universal pursuit aptitude $[6,7]$. SFLA is talented to solve separate and incessant augmentation issues. SFLA is centered on the food pursuit manners of frogs. The collaborating manners and interchange of data by the frogs while exploring for food kept on stones in a pond is exhibited. As it merges the benefits of the imitated culture process and the group's manners, it is categorized with easy application besides decent universal pursuit ability. SFLA process has been applied in numerous niches to competently solve augmentation issues.

\section{Literature Survey}

Yadav\&Yadav [8] appraised the key plan disputes centered on the prototypical of cable-less radar grids: structure-free and organized for data gathering and accumulation wherein the part of grouping and directing was deliberated for saving drive and improving the grid's lifespan. These plan approaches were the basis of any interacting convention from the drive's conservation opinion. A complete tabular summary of diverse strategies under structurefree and structured cable-less radar grids for data gathering and combination, grouping and directing was offered with key issues. Chen et al., [4] proposed a protected topographic convention of WSN which is faith-alert and of lesser drive intake called TLES. The TLES think through the faith's worth as a vital aspect disturbing the manners of nodule. In detail, the TLES would accept faith's worth, leftover drive of the nodules and nodule thickness into deliberation while choosing group's chief nodules. TLES builds these group's chief nodules by selecting the following step nodule as per expanse to base station (BS), nodules' magnitudes and leftover drive so as to create a harmless, trustworthy and drive conserving grid. Investigational outcomes reveal that the process can efficiently separate the malevolent nodule in the grid and minimize the drive's intake of the entire grid.

Zhong et al., [9] dealt with the spiteful manners of the collectors and the spiteful direction manners of the nodules in the cable-less radar grids, the reliable worth and leftover drive for the nodules to choose the collectors. A moderately dependable pathway was discretely chosen to shift the data collection outcomes. A protected grouping and dependable split multi-pathway direction detection process was proposed by the purposeful faith-based protected data collection process. The replicated investigational outcomes in NS2 program shows that the suggested process can minimize the bearing of the spiteful nodules on the grids and enhance the consistency of the cable-less radar grids.

Robinson et al., [10] proposed aEnergy Aware Clustering using Neuro-fuzzy approach (EACNF) to create the best and drive alert groups. The projected arrangement comprises ambiguous subsystem and nerve-related grid system that accomplished drive adeptness in making groups and group's chiefs in WSN. EACNF applied nerve-related grid that provides actual learning set linked to drive and thickness of all the nodules to calculate the anticipated drive for ambiguous group's chiefs. Radar nodules with greater drive were educated with several places of base station to choose drive alert group's chiefs. Ambiguous if-then charting rule was used in ambiguous reasoning part that efforts to create groups and group's chiefs. EACNF was planned for WSN that handles faith factor for safety to the grid. EACNF applied 3 metrics such as communication array, leftover drive and faith factor to improve the network's lifespan. The projected arrangement EACNF was contrasted with linked grouping arrangements, namely, Group-Chief Voting Process using Ambiguous Judgement and Drive-Alert Ambiguous Uneven Grouping. The analysis' outcomes revealed that EACNF fared superior to the other connected arrangements.

Over the years, numerous strategies and processes were surveyed for augmenting drive norm in cable-less radar grids. Directing is one among these areas wherein attempts were made for competent exploitation of drive. These endeavors used fixed (crisp) systems for creating drive-alert directing conclusions. Haider\& Yusuf [11] offered a comprehensive ambiguous judgement-based strategy for drive-alert directing in cable-less radar grids. This comprehensive strategy is lax and adjustable and hence, it can put up radar grids comprising divergent types of radar nodules with divergent drive systems.

Anita et al., [5] suggested an Fuzzy-Based Trust Prediction model for Routing (FTPR) in WSNs with minimum expenses with regard to remembrance and drive's intake. FTPR integrates a reliance forecast model that forecasts the imminent manners of the fellow citizen positioned on the historic manners, variations in reliance worth over a passé of time and endorsement discrepancy. To minimize and regulate the expenses, FTPR received endorsements from a subsection of fellow citizens who had extreme number of exchanges with the petitioner. Academic study and imitation outcomes of FTPR convention demonstrates greater pack distribution proportion, greater grid's lifespan, lesser end-to-end postponement besides lesser remembrance and drive's intake than the outdated and current reliance-centered directing arrangements. Prabaharan \& Ponnusamy [12] used the 3-select altered ACO with high importance towards mining. The 3 -select altered ACO works by recognizing the straight pathway post recognition by each ant in the metric's total pathway. . Simulated Annealing (SA) was applied to recognize the finest pathway from these accessible set of pathways. The significant attention of this strategy is to get a balance point for the finest competence and phase. Investigations were carried out applying numerous datasets and the competence and uncertainty noticed in the gained pathways showed that the process performed successfully.

Dongyao et al., [13] projected an adjustable multi-pathways strategy centered on an enhanced spring procedure to answer the communication-blocking issue in WSNs. Precisely, this paper begins with a pathway-gratification model that assumes the forecasted magnitude of blocking, the leftover drive besides the least number of stages. The process was restructured separately during the native augmentation course and an adjustable training aspect was presented. The memetic data of separate frogs was augmented with an inception-choice strategy that cheers jumping from frailer persons to better ones. In universal augmentation, the pursuit alignment of each memeplex is centered on bartering and remerging the data with other meme lexes besides a multipathway directing inkling was presented to choose the optimum pathway. Imitation outcomes reveal that this process fare good in real time, considerably enhances the drive's effectiveness besides prolonging the grid's lifespan.

Malarvizhi\&Gopi Sami Nathan [14] suggested Fuzzy Based Trust Computing Model for WSNs (FBTCM). The entire network was split into small groups and for each group, one group's chief was selected. Each group's chief calculates the straight reliance of their group's associates. For calculating the reliance of every radar communique, data and drive strictures were used. Ambiguous sensibleness was applied to merge the reliance's strictures and found the trustworthy nodules over ambiguous rules. High reliance nodules were used to be the part of inter group communique.

NS-2 centered imitation outcomes specify that the grid's function of FBTCM enhances in contrast to the current EDTM reliance prototypical convention.

Amiri et al., [15] brought out an optimum directing convention for WSN enthused by the searching manners of ants. The ants attempt to locate current pathways between the origin and the base station. Besides, this manners of ants were merged with ambiguous sensibleness in order that the ants make the finest conclusion. In other words, the ambiguous sensibleness was used to make use of these optimum pathways

The proposed process applies the doctrine of the ambiguous Fuzzy Ant Colony Optimization Routing (FACOR) to progress an appropriate solution for the problem. The function of the planned directing process was assessed by Network Simulant 2 (NS2). The 
reproduction outcomes reveal that the planned process augments the drive's intake amount, reduces the figure of directing entreaty packs and enhances the grid's lifespan in contrast with the unique AODV.

\section{Methodology}

\section{Directed Random Propagation (Drp)}

DRP [16] enhances the circulation competency by applying twostep vicinity data. More explicitly, DRP includes a "last-hop neighbor list" (LHNL) field to the legend of each share. Before a share is circulated to the next nodule, the transmitting nodule initially substitutes the old matter in the LHNL field of the share by its vicinity list. When the next nodule takes the share, it contrasts the LHNL field against its own vicinity list and arbitrarily takes one nodule from its vicinity that were absent in the LHNL. It then reduces the TTL's importance, rekindles the LHNL field and transmits the share to the next step, etc. Whenever the LHNL fully overlays with or encloses the transmitting nodule's close by inhabitants' list, its random is drawn, just like in PRP scheme. In accordance to this proliferation method, DRP minimizes the likelihood of proliferating a share back and forth by removing this type of proliferation in the interior of any two instantaneous successive stages. Contrasted with PRP, DRP tries to thrust a share noticeably away from the origin and thus taking to improved proliferation competency for an assumed TTL significance.

\section{Fuzzy Trust}

Figure 1 reveals the purposeful block illustration for ambiguous sensible centered reliance calculation. It has vaguer, vague engine, vague rule besides clearer modules. Fuzzier adapts the crunchy data from communique's reliance, drive's reliance and data's reliance into vague values like low, medium and high. Vague rule is formed centered on the merger of response stricture as appears in Table 1. Vague machine checks the responses with vague rule and produces the yields. Clearer produces end reliance's significance. Contrasted with other reliance calculating processes, vague sensibleness creates additional summarized yield [14].

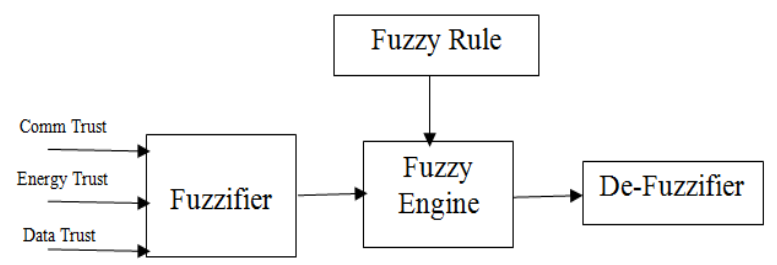

Figure 1: Functional block diagram of fuzzy trust computation

Table 1: Fuzzy Rule

\begin{tabular}{|c|c|c|c|}
\multicolumn{7}{|c|}{ Table 1: Fuzzy Rule } \\
\hline COM Trust & Energy Trust & Data Trust & Direct Trust \\
\hline High & High & High & High \\
\hline High & High & Medium & High \\
\hline High & Medium & Medium & High \\
\hline Medium & Medium & Low & Medium \\
\hline$\ldots$ & $\ldots$ & $\ldots$ & $\ldots$ \\
\hline Low & Low & Low & Low \\
\hline
\end{tabular}

\section{Shuffled Frog Leaping Algorithm (Sfla)}

The SFLA is a populace-centered empirical pursuit with a merger of deterministic and arbitrary approaches. The deterministic approach guarantees robust empirical pursuit of the process whereas the arbitrary approach permits elasticity besides sturdiness of the pursuit course [17]. The SFLA comprises meme lexes which is a set of simulated frogs. The jumping attitude of the frog improves its function. Frogs in each memeplex signify divergent answer and its quality can be applied to alter other frogs.
In the imitating progress step, information is swapped among the frogs in a memeplex [18]. The frogs are shambled post a clear figure of imitating progress step for enhanced standard of memeplex. Shambling lifts the meme's standard besides ensuring social growth. The imitating progress and shambling are reiterated until conjunction is achieved.

The steps in SFLA include [19]:

- Early inhabitants: Frogs signifying explanations are arbitrarily primed. In this work, each frog signifies a dualistic encrypted answer with ' 0 ' signifying the nodule not chosen as $\mathrm{CH}$ and ' 1 ' signifying a $\mathrm{CH}$. Each key guarantees that all nodules are concealed in the grid.

- Arranging and dispersal: Frogs are graded centered on their aptness standards and are clustered into $\mathrm{m}$ meme lexes with $n$ frogs.

- Memeplex Progress: Local pursuit is carried out.

- Shambling: Frogs are shambled among the meme lexes to safeguard enhancement in the excellence and to shift towards optimum answer.

- Terminal status: The process ceases post fixed figure of reiterations or desired inception is received.

- Shambled Frog Jumping Process is explained in various paces as hereunder:

- $\quad$ Step 1: The shambled Frog jumping process involves an inhabitant ' $\mathrm{P}$ ' of viable answer, described by a group of practical frogs $(n)$.

- Step 2: frogs are arranged in downward order in accordance with their aptness and then segregated into subsections called as meme lexes (m).

- Step 3: frogs i is articulated as $X_{i}=\left(X_{i 1}, X_{i 2}, \ldots, X_{i s}\right)$ where $\mathrm{S}$ signifies the numeral of inconsistencies.

- Step 4: within each memeplex, the frog with nastiest and best aptness are recognized as $X_{w}$ and $X_{b}$.

- Step 5: Frog with universal best aptness is identified as $X_{g}$.

- $\quad$ Step 6: The frog with nastiest aptness is enhanced in accordance with the under mentioned equation.

$$
\begin{aligned}
& D_{i}=\operatorname{rand}()\left(\mathrm{X}_{b}-X_{w}\right) \\
& X_{\text {neww }}=X_{\text {oldw }}+D_{i}\left(-D_{\text {max }} \leq D_{i} \leq D_{\text {max }}\right)
\end{aligned}
$$

(1)

where rand is an arbitrary number in the range of $[0,1]$. Di is the frog jumping pace dimension of the ith frog and $D_{\max }$ is the greatest pace permissible alteration in a frog's status. If the aptness significance of new $X_{w}$ is improved over the existing one, $X_{w}$ will be adopted; else, then the computed () is recurrent with $\mathrm{X}_{b}$ substituted by $\mathrm{X}_{g}$. If no enhancement turn out to be possible in the case, a new $X_{w}$ will be created arbitrarily. Reprise the apprise operation for a specific number of reiterations.

\section{Results and Discussion}

For experiment, fuzzy trust energy aware DRP and SFLA- fuzzy trust energy aware DRP are applied. Table 2 to 4 and figure 2 to 4 shows the Interception probability, average end to end delay and average packet loss rate respectively. 
Table 2: Interception Probability for SFLA-FTEA DRP

\begin{tabular}{|c|c|c|}
\hline $\begin{array}{c}\text { Number of } \\
\text { nodes }\end{array}$ & $\begin{array}{c}\text { Fuzzy Trust Energy } \\
\text { Aware DRP }\end{array}$ & $\begin{array}{c}\text { SFLA-Fuzzy Trust Energy } \\
\text { Aware DRP }\end{array}$ \\
\hline 100 & 0.16 & 0.12 \\
\hline 200 & 0.13 & 0.09 \\
\hline 300 & 0.12 & 0.07 \\
\hline 400 & 0.09 & 0.06 \\
\hline 500 & 0.07 & 0.04 \\
\hline 600 & 0.06 & 0.02 \\
\hline
\end{tabular}

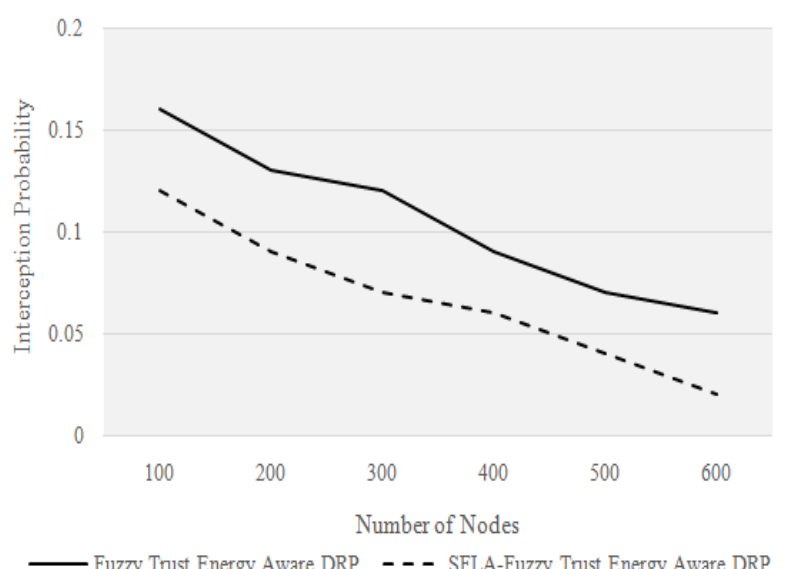

Figure 2: Interception probability for SFLA-FTEA DRP

It is observed from table 2 and figure 2 that the interception probability for SFLA-Fuzzy Trust Energy Aware DRP performs better by lowering its value by $28.57 \%, 52.63 \%$ and $100 \%$ than Fuzzy Trust Energy Aware DRP at number of nodes 100, 300 and 600 respectively.[20]

Table 3: Average End to End Delay for SFLA-FTEA DRP

\begin{tabular}{|c|c|c|}
\hline Number of nodes & $\begin{array}{c}\text { Fuzzy Trust } \\
\text { Energy Aware } \\
\text { DRP }\end{array}$ & $\begin{array}{c}\text { SFLA-Fuzzy Trust } \\
\text { Energy Aware DRP }\end{array}$ \\
\hline 100 & 0.00161 & 0.00152 \\
\hline 200 & 0.00172 & 0.00163 \\
\hline 300 & 0.01784 & 0.01648 \\
\hline 400 & 0.03055 & 0.02889 \\
\hline 500 & 0.0619 & 0.05849 \\
\hline 600 & 0.06835 & 0.06403 \\
\hline
\end{tabular}

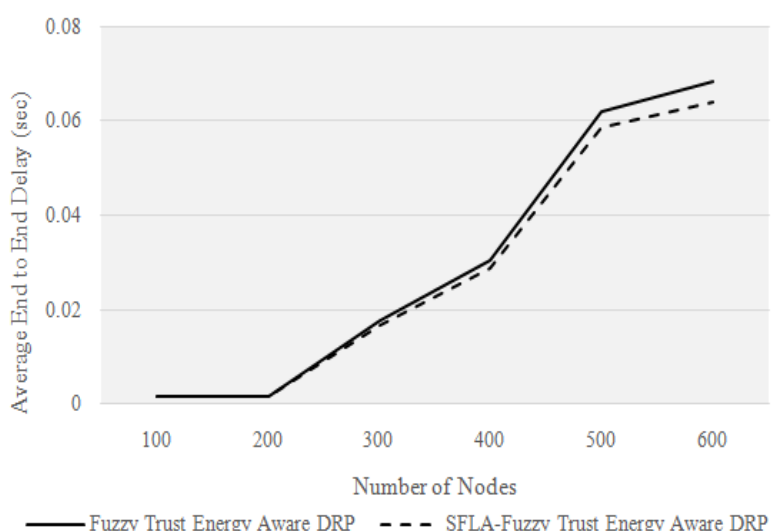

Figure 3: Average End to End Delay for SFLA-FTEA DRP

It is observed from table 3 and figure 3 that the Average end to end delay for SFLA-Fuzzy Trust Energy Aware DRP performs better by lowering its value by $5.75 \%, 7.93 \%$ and $6.53 \%$ than Fuzzy Trust Energy Aware DRP at number of nodes 100, 300 and 600 respectively.
Table 4: Average Packet Loss Rate for SFLA-FTEA DRP

\begin{tabular}{|c|c|c|}
\hline $\begin{array}{c}\text { Number of } \\
\text { nodes }\end{array}$ & $\begin{array}{c}\text { Fuzzy Trust Energy } \\
\text { Aware DRP }\end{array}$ & $\begin{array}{c}\text { SFLA-Fuzzy Trust Energy } \\
\text { Aware DRP }\end{array}$ \\
\hline 100 & 6.91 & 6.41 \\
\hline 200 & 10.74 & 10.17 \\
\hline 300 & 10.57 & 9.94 \\
\hline 400 & 16.04 & 15.29 \\
\hline 500 & 20.34 & 19.49 \\
\hline 600 & 24.71 & 23.29 \\
\hline
\end{tabular}

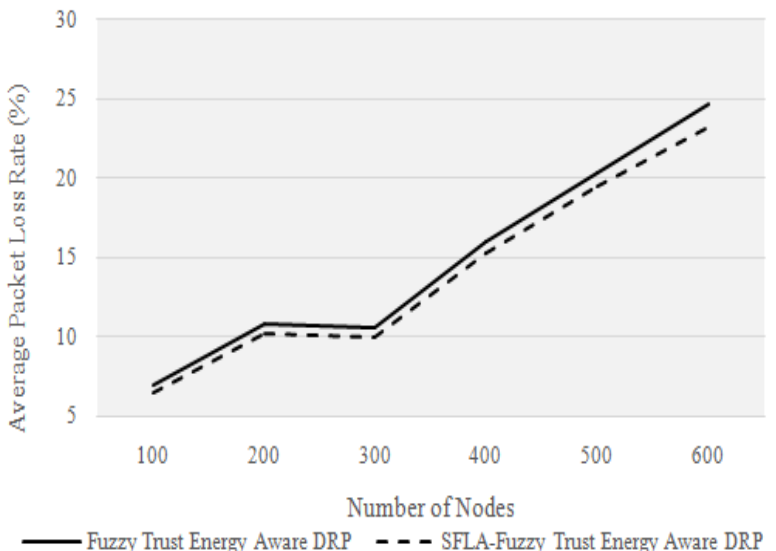

Figure 4: Average Packet Loss Rate for SFLA-FTEA DRP

It is observed from table 4 and figure 4 that the Average packet loss rate for SFLA-Fuzzy Trust Energy Aware DRP performs better by lowering its value by $7.51 \%, 6.14 \%$ and $5.92 \%$ than Fuzzy Trust Energy Aware DRP at number of nodes 100, 300 and 600 respectively.[21]

\section{Conclusion}

Cable-less Radar Grids (WSNs) can be securely renowned as one among the most significant expertise since recently. Due to certain constraints in radar nodules like restricted electrical energy backup source, certain frequently applied processes like directing, optimum planning and competent process seems to be compulsory. Trust model is an efficient approach to repel diverse attacks in the radar grids. The outcomes reveal that the capture likelihood for SFLA-vague reliance Drive Alert DRP fares great by lessening its worth by $28.57 \%, 52.63 \%$ and $100 \%$ over Reliance Drive Alert DRP at a figure of nodules 100, 300 and 600 respectively. The average end to end holdup for SFLA-vague reliance drive alert DRP fares great by minimizing its value by $5.75 \%, 7.93 \%$ and $6.53 \%$ over vague reliance drive alert DRP at a figure of nodules 100, 300 and 600 respectively. Besides the average pack loss rate for SFLA-vague reliance drive alert DRP fares great by minimizing its value by $7.51 \%, 6.14 \%$ and $5.92 \%$ over vague reliance drive alert DRP at a figure of nodules 100 , 300 and 600 respectively..

\section{References}

[1] Ramesh R \& Varshney PK, "Data aggregation techniques in sensor networks: A survey", Electrical Engineering and Computer Science. Paper, Vol.22, (2006)

[2] Amiri E, Keshavarz H, Heidari H, Mohamadi E \& Moradzadeh H, "Intrusion detection systems in MANET: a review", ProcediaSocial and Behavioral Sciences, Vol.129, (2014), pp.453-459.

[3] Keshavarz H, Noor RM \& Mostajeran E, "Using routing table flag to improve performance of AODV routing protocol for VANETs environment", 9th International Conference on Computing and Information Technology (IC2IT2013), (2013), pp.73-82. 
[4] Chen Z, He M, Liang W \& Chen K, "Trust-aware and low energy consumption security topology protocol of wireless sensor network", Journal of Sensors, (2015).

[5] Anita X, Bhagyaveni MA \& Manickam J, "Fuzzy-based trus prediction model for routing in WSNs", The Scientific World Journal, (2014).

[6] Chen K, "Unequal cluster-based routing protocol in wireless sensor networks", Journal of Net- works, Vol.8, No.11, (2013), pp.2656-2662.

[7] Binitha S \& Sathya SS, "A survey of bio inspired optimization algorithms", International Journal of Soft Computing and Engineering, Vol.2, No.2, (2012), pp.137-151.

[8] Yadav S \& Yadav RS, "A review on energy efficient protocols in wireless sensor networks", Wireless Networks, Vol.22, No.1, (2016), pp.335-350.

[9] Zhong C, Mo Y, Zhao J, Lin C \& Lu X, "Secure clustering and reliable multi-path route discovering in wireless sensor networks", IEEE Sixth International Symposium on Parallel Architectures, Algorithms and Programming (PAAP), (2014), pp.130-134.

[10] Robinson YH, Julie EG, Balaji S \& Ayyasamy A, "Energy Aware Clustering Scheme in Wireless Sensor Network Using Neuro-Fuzzy Approach", Wireless Personal Communications, (2016), pp.1-19.

[11] Haider T \& Yusuf M, "A fuzzy approach to energy optimized routing for wireless sensor networks", Int. Arab J. Inf. Technol., Vol.6, No.2, (2009), pp.179-185.

[12] Prabaharan SB \& Ponnusamy R, "Energy Aware Secure Dynamic Multipath Routing using ACO with Modified Local Selection using SA", Energy, Vol.3, No.5, (2016).

[13] Dongyao J, Shengxiong Z, Meng L \& Huaihua Z, "Adaptive multipath routing based on an improved leapfrog algorithm", Information Sciences, Vol.367, (2016), pp.615-629.

[14] Malarvizhi M \& GopiSaminathan A, "Fuzzy Based Trust Computing Model For Wireless Sensor Network", International Journal of Emerging Technology in Computer Science \& Electronics (IJETCSE), Vol.22, No.2, (2016).

[15] Amiri E, Keshavarz H, Alizadeh M, Zamani M \& Khodadadi T, "Energy efficient routing in wireless sensor networks based on fuzzy ant colony optimization", International Journal of Distributed Sensor Networks, (2014).

[16] Shu T, Krunz M \& Liu S, "Secure data collection in wireless sensor networks using randomized dispersive routes", IEEE transactions on mobile computing, Vol.9, No.7, (2010), pp.941-954.

[17] Roy P \& Chakrabarti A, "Implementation of genetic algorithm and modified shuffled frog leaping algorithm for transmission loss minimum re-scheduling", Annual IEEE India conference (INDICON), 2011, pp.1-4.

[18] Anandamurugan S \& Abirami T, "Antipredator adaptation shuffled frog leap algorithm to improve network life time in wireless sensor network", Wireless Personal Communications, (2016), pp.1-12.

[19] Zhang X, Hu X, Cui G, Wang Y \& Niu Y, “An improved shuffled frog leaping algorithm with cognitive behavior", Proc. 7th world congr. Intelligent Control and Automation, (2008).

[20] Akhpanov, S. Sabitov, R. Shaykhadenov (2018). Criminal pre-trial proceedings in the Republic of Kazakhstan: Trend of the institutional transformations. Opción, Año 33. 107-125.

[21] Z Iskakova, M Sarsembayev, Z Kakenova (2018). Can Central Asia be integrated as asean? Opción, Año 33. 152-169. 